\title{
Interação salinidade da água de irrigação e substratos na produção de mudas de maracujazeiro amarelo
}

\author{
Fernanda Andrade de Oliveira', Maria Ângela Casimiro Lopes', Francisco Vanies da Silva Sá ${ }^{2 *}$, \\ Reginaldo Gomes Nobre', Romulo Carantino Lucena Moreira', \\ Luderlândio de Andrade Silva', Emanoela Pereira de Paiva²
}

\section{Resumo}

Objetivou-se avaliar a produção de mudas de maracujazeiro amarelo em resposta a interação entre a composição de substratos e níveis de salinidade da água de irrigação em experimento desenvolvido no período de novembro de 2013 a janeiro de 2014, em ambiente protegido (casa de vegetação) do Centro de Ciências e Tecnologia Agroalimentar - CCTA, da Universidade Federal de Campina Grande - UFCG, localizado no município de Pombal, Paraíba PB. Adotandose $o$ delineamento experimental de blocos casualizados, em esquema fatorial $4 \times 3$ constituído de quatro níveis de salinidade da água de irrigação - CEa $\left(0,3 ; 1,5 ; 2,5\right.$ e 3,5 dS $\left.\mathrm{m}^{-1}\right)$ e três substratos [S= solo; $\mathrm{S}+\mathrm{EO}=$ solo + esterco ovino (2:1);e $\mathrm{S}+\mathrm{SC}=$ solo + substrato comercial (2:1)], totalizando 12 tratamentos, com três repetições e três plantas uteis por repetição. O aumento da salinidade da água de irrigação reduziu a emergência, o crescimento e o acúmulo de matéria seca das mudas de maracujazeiro amarelo, sendo a matéria seca total a variável mais afetada pela salinidade. $O$ substrato contendosolo + esterco ovino na proporção (2:1) promoveu o maior crescimento inicial e acúmulo de matéria seca as mudas de maracujazeiro amarelo quando irrigadas com salinidade até o nível de $1,5 \mathrm{dS} \mathrm{m}^{-1}$.

Palavras-chave: crescimento inicial, estresse salino, Passiflora edulis f. flavicarpa Deg.

\section{Interaction of irrigation water salinity and substrate on the production of yellow passion fruit seedlings}

\begin{abstract}
The aim of the present study was to evaluate the yield og yellow passion fruit seedling according to the interaction between substrate composition and salinity levels of irrigation water in an experiment carried out from November, 2013 to January 2014, in a protected environment (greenhouse) at the Center of Science and Agrifood Technology - CCTA, Federal University of Campina Grande - UFCG, located in the municipality of Pombal, PB, Brazil. A randomized complete block design in a $4 \times 3$ factorial consisted of four levels of irrigation water salinity ECW $\left(0.3,1.5,2.5\right.$ and $\left.3.5 \mathrm{dS} \mathrm{m}^{-1}\right)$ and three different substrates $[S=$ soil; $E O=$ Soil + sheep manure (2:1); and SC = soil + commercial substrate $(2: 1)$ ], with a total of 12 treatments with three replications and three useful plants per replication were used. The increase in salinity of irrigation water reduced the emergence, growth and dry matter accumulation of passion fruit seedlings, being the total dry matter the variable that was most affected by salinity. The substrate containing soil + sheep manure in the ratio of 2:1 promoted the highest initial growth and dry matter accumulation of yellow passion fruit seedlings, using a maximun level of $1.5 \mathrm{dS} \mathrm{m}^{-1}$ of saline irrigation were used.
\end{abstract}

Keywords: initial growth, salinity stress, Passiflora edulis f. flavicarpa Deg. 


\section{Introdução}

Em quase todos os estados brasileiros - maracujazeiro amarelo (Passiflora edulis $f$. flavicarpaDeg.) destaca-se como frutífera de expressiva importância socioeconômica. Ademais, o Brasil é o maior produtor e consumidor mundial da fruta, obtendo em 2012 uma produção de776.097 toneladas das quais 563.346 toneladas foram produzidas na região nordeste (IBGE,2012). Toda essa expressividade está associada às condições edafoclimáticas favoráveis para o seu crescimento, desenvolvimento e produção (Mendonça et al., 2006).

A crescente necessidade em se aumentar a produção de alimentos tem requerido a expansão das áreas cultivadas. Sendo que, essa busca não leva em conta apenas a incorporação das áreas agrícolas consideradas adequadas ao cultivo, mas, também, o aproveitamento de áreas degradadas, como os solos afetados por sais, e uso de águas consideradas de qualidade inferior, como as de elevados teores salinos (Banardet al., 2010).Esta situação é mais evidente nas áreas semiáridas do Brasil, em que as águas utilizadas nas irrigações, quase sempre, possuem concentrações salinas que podem comprometero solo e principalmente o desenvolvimento das culturas, sendo esse mais intenso na fase inicial da grande maioria das fruteiras (Sá et al., 2013; Brito et al., 2014).

No caso do maracujazeiro, a adversidade revela-se ainda mais agressiva devido à cultura ser sensível à salinidade da água ou do solo (Ayers \& Westcot, 1999). Os efeitos mais marcantes da salinidade sobre as plantas se refletem em alterações no potencial osmótico, na toxicidade iônica e no desequilíbrio nutricional, provocando a redução do seu crescimento e consequentemente, sérios prejuízos à atividade agrícola (Sousa et al., 2008; Ahmed \& Montani, 2010); esse estresse ocasionado pelo manejo inadequado da água salina, podendo aumentar os teores de sódio no solo, e gradualmente a porcentagem de sódio trocável, a razão de adsorção de sódio e a condutividade elétrica do solo (Holanda Filho et al., 2011).
Além da salinidade, outro fator importante paraque se tenha um resultado satisfatório no cultivo de fruteirasé a produção de mudas de qualidade, e para que isso aconteça, um dos fatores importantes é escolha do substrato (Costaet al., 2010).Segundo Pires etal. (2008)a função do substrato é dar sustentação às plantas, proporcionar o crescimento das raízes e fornecer as quantidades adequadas de ar, água e nutrientes. Tais parâmetros são observados na germinação,e exerce grande influência sobre a emergência das plântulas e formação das mudas.

Diante disso, objetivou-se avaliar a produção de mudas de maracujazeiro amarelo em resposta a interação entre a composição do substrato e níveis de salinidade da água de irrigação.

\section{Material e Métodos}

O experimento foi desenvolvido em ambiente protegido (casa de vegetação) do Centro de Ciências e Tecnologia Agroalimentar - CCTA, da Universidade Federal de Campina Grande - UFCG, localizado no município de Pombal, Paraíba PB, nas coordenadas

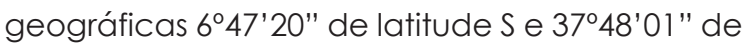
longitude $W$, a uma altitude média de 174 m;no período 11 de novembro de 2013 a 10 de janeiro de 2014.

Adotou-se o delineamento experimental de blocos casualizados, usando um esquema fatorial $4 \times 3$ constituído de quatro níveis de salinidade da água de irrigação $(0,3 ; 1,5 ; 2,5$ e $3,5 \mathrm{dS}^{-1}$ ) e três substratos [S= solo; $\mathrm{S}+\mathrm{EO}=$ solo + esterco ovino (2:1); e $\mathrm{S}+\mathrm{SC}=$ solo + substrato comercial (2:1)], totalizando 12 tratamentos, com três repetições e três plantas uteis por repetição.

As mudas foram produzidas em sacos de polietileno com capacidade para 0,5 $\mathrm{dm}^{3}$. Para a confecção dos substratos foram utilizados solos de barranco (Franco-arenoso), esterco ovino devidamente curtido com relação $\mathrm{C} / \mathrm{N}$ 12:1 e substrato comercialTropstrat ${ }^{\circledR} \mathrm{Cujas}$ características químicas (Tabela 1) foram realizadas conforme metodologia descrita por Claess em (1997).

A semeadura foi realizada de modo a distribuir três sementes por sacos de forma 
equidistante na profundidade de $1 \mathrm{~cm}$. As sementes de maracujazeiro azedo cV. BRS Gigante Amarelo, pré-dispondo de $85 \%$ de germinação. Após a estabilização da emergência aos 21 dias após a semeadura (DAS) foi realizado o desbaste, deixando apenas uma planta por recipiente.

Tabela 1. Características químicas dos componentes dos substratos usado na produção de mudas de maracujazeiro.

\begin{tabular}{|c|c|c|c|c|c|c|c|c|c|c|c|c|}
\hline & CE & $\mathrm{pH}$ & $\mathrm{P}$ & $\mathrm{K}^{+}$ & $\mathrm{Ca}^{+2}$ & $\mathrm{Mg}^{+2}$ & $\mathrm{Na}^{+}$ & $\mathrm{Al}^{l^{3+}}$ & $\mathrm{H}^{+}+\mathrm{Al}^{3+}$ & SB & $\mathrm{T}$ & MO \\
\hline & $d \mathrm{dSm}^{-1}$ & $\mathrm{H}_{2} \mathrm{O}$ & $\mathrm{mgdm}^{-3}$ & & & & $--\mathrm{cmo}$ & $\mathrm{Im}^{-3}-$ & & 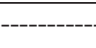 & & $\mathrm{g} \mathrm{kg}^{-3}$ \\
\hline$S$ & 0,09 & 8,07 & 3,00 & 0,32 & 6,40 & 3,20 & 0,18 & 0,00 & 0,00 & 9,92 & 9,92 & 16,0 \\
\hline EO & 6,26 & 7,75 & 56,0 & 24,64 & 7,70 & 15,90 & 9,18 & 0,00 & 0,00 & 57,42 & 57,42 & 180,0 \\
\hline SC & 1,65 & 5,75 & 6,00 & 1,67 & 11,60 & 28,50 & 17,84 & 0,00 & 11,88 & 41,77 & 41,77 & 570,0 \\
\hline
\end{tabular}

$\mathrm{SB}=$ soma de bases; $\mathrm{CE}=$ condutividade elétrica; $\mathrm{T}$ = capacidade de troca de cátions total; $\mathrm{M} . \mathrm{O}=$ matéria orgânica; $\mathrm{S}=\mathrm{Solo} ; \mathrm{EO}=$ esterco ovino; $\mathrm{SC}=$ substrato comercial.

As águas salinas foram preparadas com adição de cloreto de sódio a água de abastecimento local $\left(\mathrm{CE}_{\mathrm{a}}=0,3 \mathrm{dS} \mathrm{m}^{-1}\right) ; \mathrm{O} \mathrm{NaCl}$ é predominante aos íons presentes em fontes de água utilizada para irrigação, em pequenas propriedades do Nordeste brasileiro (Sá et al., 2013). Acrescenta-se que, para preparo das águas os sais foram pesados conforme tratamento, adicionando-se água, até ser atingido o nível desejado de CE, conferindose os valores com um condutivímetro portátil ajustado a temperatura de $25^{\circ} \mathrm{C}$.

As irrigações foram realizadas duas vezes ao dia a partir da semeadura das sementes, no início da manhã e no final da tarde, de modo a deixar o solo com umidade próxima à capacidade máxima de retenção, sendo a lâmina aplicada acrescida de uma fração de lixiviação (FL) de 20\%. O volume aplicado (Va) por recipiente era obtido pela diferença entre a lamina aplicada no dia anterior (La) menos a média de drenagem (D), dividido pelo número de recipientes (n), como indicado na equação 1, abaixo:

$$
V a=\frac{L a-D}{n(1-F L)}
$$

Aos 30 DAS foram avaliadas a percentagem de emergência (PE), correlacionando números de plântulas emergidas em relação ao número de sementes semeadas e os dias paraemergência (DPE), com base na equação 2 , descrita em Lima et al. (2006).

$D P E=\frac{\left(N_{1} G_{1}\right)+\left(N_{2} G_{2}\right)+\cdots+\left(N_{n} G_{n}\right)}{G_{1}+G_{2}+\cdots+G_{n}}$
Onde:

$\mathrm{N} 1$ = número de dias para a primeira contagem; Gl = número de plântulas emergidas na primeira contagem;

N2 = número de dias para a segunda contagem; G2 = número de plântulas emergidas na segunda contagem;

$\mathrm{Nn}$ = número de dias para a última contagem;

Gn = número de plântulas emergidas na última contagem.

Aos 60 DAS foram aferidas a altura das plantas $(\mathrm{cm})$ com o auxilio de uma régua graduada da base até o ápice, o diâmetro do caule $(\mathrm{mm})$ a $1 \mathrm{~cm}$ do solo com auxílio de paquímetro digital e o número de folhas, por meio da contagem das folhas totalmente abertas. Após a obtenção desses dados as plantas foram cortadas rentes ao solo, realizando a partição da parte aérea e das raízes, sendo em seguida, acondicionadas em sacos de papel devidamente identificados conforme tratamento, e colocadas para secar em estufa de circulação de ar a $65^{\circ} \mathrm{C}$ até atingirem massa constante.De posse desses dados, determinouse a matéria seca da parte aérea (MSPA), a matéria seca da raiz (MSR) e matéria seca total (MST= MSPA+MSR).

As variáveis analisadas foram submetidas à análise de variância (teste F), quando significativas procedeu-se o teste de Tukey até $5 \%$ de probabilidade paras as características qualitativas, e análise de regressão polinomial para os dados quantitativos utilizando-se do software Sisvar (Ferreira, 2011). 


\section{Resultados e Discussão}

Observaram-se interações significativas entre a salinidade da água de irrigação e os substratos para todas as variáveis analisadas, com exceção das variáveis DPE e DC que foram influenciadas significativamente pelos dois fatores isolados ao nível de $1 \%$ de probabilidade (Tabela 2). Tais resultados indicam que a escolha do substrato influencia sob os efeitos da salinidade em plantas de maracujazeiro amarelo, desse modo, podendo viabilizar a produção de mudas da cultura mesmo quando irrigadas com água salina.

A emergência das plântulas de maracujazeiro amarelo foi reduzida pelo aumento da salinidade da água de irrigação, sendo que as plantas cultivadas apenas com solo como substrato necessitaram de um o maior números de dias para emergir (17,92 dias) em relação aos demais substratos sob o maior nível de salinidade estudado $\left(3,5 \mathrm{dS} \mathrm{m}^{-1}\right)$, que não diferiram entre si (Tabela 3). Possivelmente, a não adição de matéria orgânica ao substrato tenha reduzindo a emergência das plantas, já que esta favorece a aeração e a retenção de água no solo.

Observou-se efeito significativo da interação salinidade da água de irrigação versus substratos, principalmente atribuída aos níveis de salinidade já que os mesmos também influenciaram significativamente percentagem de emergência das plântulas de maracujazeiro (Tabela 2 e 3).

Tabela 2. Resumo da análise de variância das variáveis dias para emergência (DPE), percentagem de emergência (PE) (\%), altura de planta (AP) (cm), diâmetro caulinar (DC) (mm), número de folhas (NF), matéria seca da parte aérea (MSPA), da raiz (MSR) e total (MST) de mudas de maracujazeiro amarelo em função substratos (SUB) e salinidade da água de irrigação (SAL).

\begin{tabular}{cccccccccc}
\hline \multirow{2}{*}{ FV } & \multirow{2}{*}{ GL } & \multicolumn{10}{c}{ Quadrado Médio } \\
\cline { 3 - 9 } & & DPE & PE & AP & DC & NF & MSPA & MSR & MST \\
\hline SAL & 3 & $17,68^{* *}$ & $1171,32^{* *}$ & $15,99^{* *}$ & $0,16^{* *}$ & $10,88^{* *}$ & $0,065^{* *}$ & $0,030^{* *}$ & $0,1835^{* *}$ \\
SUB & 2 & $7,90^{* *}$ & $179,67^{\text {NS }}$ & $2,17^{*}$ & $0,81^{* *}$ & $13,25^{* *}$ & $0,314^{* *}$ & $0,050^{* *}$ & $0,6164^{* *}$ \\
SAL x SUB & 6 & $0,83^{\text {NS }}$ & $965,23^{* *}$ & $1,37^{*}$ & $0,07^{\text {NS }}$ & $1,90^{*}$ & $0,050^{* *}$ & $0,018^{* *}$ & $0,1288^{* * *}$ \\
BLOCO & 2 & $1,10^{\text {NS }}$ & $9,91^{\text {NS }}$ & $0,47^{\text {NS }}$ & $0,05^{\text {NS }}$ & $0,35^{\text {NS }}$ & $0,002^{\text {NS }}$ & $0,001^{\text {NS }}$ & $0,0002^{\text {NS }}$ \\
ERRO & 22 & 0,92 & 93,34 & 0,49 & 0,04 & 0,64 & 0,003 & 0,001 & 0,0046 \\
\hline CV & & 6,50 & 14,18 & 10,73 & 12,76 & 18,28 & 34,71 & 60,69 & 28,81 \\
\hline$* *=1 \%$
\end{tabular}

Os melhores resultados para percentagem de emergência foram obtidos no com CEa de 1,5 dSm-1 onde os substratos não diferiram estatisticamente (Tabela 3). Observa-se ainda que no maior nível de salinidade, houve diferença apenas em relação ao S+SC que teve maior PE $(74,07)$ (Tabela 3).

A irrigação com água salina aumenta diretamente os teores de sais no substrato, principalmente os sais de sódio, esses sais em altas concentrações promovem interações hormonais às plantas (Taiz \& Zaiger, 2013), além de reduzir o potencial osmótico do substrato, que possivelmente ao atuarsob a germinação e emergência prejudique 0 embebição da semente prejudicando o crescimento embrionário, limitando com isso o processo de emergências das plântulas. Tais resultados corroboram com os observado por Araújo et al. (2013) em que o aumento da salinidade da água de irrigação reduziu a percentagem de emergência em plantas de maracujazeiro amarelo.

Resultados semelhantes também são verificados em outras frutíferas a exemplo do Cajueiro (Sousa et al., 2011 ) e mamoeiro (Sá et al., 2013), verificando-se reduções na emergência de plantas conforme o aumento da salinidade da água de irrigação.

A salinidade da água de irrigação reduziu em 37,0\%; $13,8 \%$ e $42,3 \%$ a altura, o diâmetro do caule e número de folhasdas mudas de maracujazeiro amarelo respectivamente quando comparados as plantas sob menor CEa $\left(0,3 \mathrm{dS} \mathrm{m}^{-1}\right)$ em relação ao maiorCEa $\left(3,5 \mathrm{dS} \mathrm{m}^{-1}\right)$ em média para os substrato utilizados (Tabela 4). No entanto, o substrato $S+E O$ proporcionou $O$ maior crescimento em altura, diâmetro do caule e número de folhas das mudas de maracujazeiro amarelo até o nível de 1,5 dS $\mathrm{m}^{-1}$, entretanto, a 
partir desse nível não foi verificado diferenças significativas no crescimento das mudas sob distintos substratos (Tabela 4). Foi verificado que a partir desse nível o aumente da concentração de sais de sódio no substrato exerceu efeito de fitotoxicidade nas plantas de maracujazeiro desencadeando um conjunto de alterações iônicas e hormonais afetando diretamente o crescimento das plantas (Taiz \& Zaiger, 2013; Sá et al., 2013).

Observa-se ainda que a maior redução foi no número de folhas, o que possivelmente pode estar relacionado ao mecanismo de tolerância da espécie em reduzir a área foliar, de modo a minimizar a atividade transpiratória e com isso absorção de água e sais tóxicos pela planta, reduzindo o efeito da toxicidade por íons específicos (Flowers\&Flowers, 2005).

Reduções no crescimento de mudas de maracujazeiro amarelo irrigadas com água salina também foram verificadas por Cavalcante et al. (2009) e por Araújo et al. (2013), esse fenômeno é observado no crescimento inicial de outras frutíferas em função do aumento da salinidade da água de irrigação, a exemplo da aceroleira (Gurgel et at., 2007), em porta enxertos de citros (Fernandes et al., 2011) e em mudas de mamoeiro (Sá et al., 2013).

Tabela 3. Teste de médias e analise de regressão das variáveis dias para emergência e percentagem de emergência de plântulas de maracujazeiro amarelo em função substratos (SUB) e salinidade da água de irrigação (SAL) aos 21 dias após a semeadura.

\begin{tabular}{|c|c|c|c|c|c|c|}
\hline \multicolumn{7}{|c|}{ Dias para Emergência (Dias) } \\
\hline \multirow{2}{*}{ Substratos } & \multicolumn{6}{|c|}{ Salinidade da Água $\left(\mathrm{dS} \mathrm{m}^{-1}\right)$} \\
\hline & 0,3 & 1,5 & 2,5 & 3,5 & Regressão & $\mathrm{R}^{2}$ \\
\hline S & $13,76 \mathrm{~A}$ & $15,3 \mathrm{~A}$ & $15,78 \mathrm{~A}$ & $17,92 \mathrm{~A}$ & $\hat{Y}=13,315+1,2177 x$ & $0,94^{* *}$ \\
\hline$S+E O$ & $12,3 \mathrm{~A}$ & $13,95 \mathrm{~A}$ & $15,22 \mathrm{~A}$ & $15,58 \mathrm{~B}$ & $\hat{Y}=12,199+1,0581 x$ & $0,95^{* *}$ \\
\hline$S+S C$ & $12,75 \mathrm{~A}$ & $14,68 \mathrm{~A}$ & $14,33 \mathrm{~A}$ & $15,64 \mathrm{~B}$ & $\hat{Y}=12,85+0,7463 x$ & $0,80 * *$ \\
\hline \multicolumn{7}{|c|}{ Percentagem de Emergência (\%) } \\
\hline \multirow{2}{*}{ Substratos } & \multicolumn{6}{|c|}{ Salinidade da Água (dS m $\left.{ }^{-1}\right)$} \\
\hline & 0,3 & 1,5 & 2,5 & 3,5 & Regressão & $\mathrm{R}^{2}$ \\
\hline $\mathrm{S}$ & $77,78 \mathrm{~A}$ & $85,19 \mathrm{~A}$ & $62,96 \mathrm{~A}$ & $44,44 \mathrm{~B}$ & $\hat{Y}=89,468-11,218 x$ & $0,72^{* *}$ \\
\hline$S+E O$ & $70,37 \mathrm{AB}$ & $92,59 \mathrm{~A}$ & $43,33 \mathrm{~A}$ & $51,85 \mathrm{~B}$ & $\hat{Y}=83,093-9,5159 x$ & $0,35^{* *}$ \\
\hline$S+S C$ & 51,85 B & $74,07 \mathrm{~A}$ & $88,89 \mathrm{~A}$ & $74,07 \mathrm{~A}$ & $\hat{Y}=40,427+36,482 x-7,5516 x^{2}$ & $0,94^{* *}$ \\
\hline
\end{tabular}

Tabela 4. Teste de médias e analise de regressão das variáveis alturade plantas (AP) (cm), diâmetro caulinar (DC) $(\mathrm{mm})$ e número de folhas (NF) de mudas de maracujazeiro amarelo em função substratos (SUB) e salinidade da água de irrigação (SAL) aos 60 dias após a semeadura.

\begin{tabular}{|c|c|c|c|c|c|c|}
\hline \multicolumn{7}{|c|}{ Altura $(\mathrm{cm})$} \\
\hline \multirow{2}{*}{ Substratos } & \multicolumn{6}{|c|}{ Salinidade da Água (dS m-1) } \\
\hline & 0,3 & 1,5 & 2,5 & 3,5 & Regressão & $\mathrm{R}^{2}$ \\
\hline$S$ & $7,96 \mathrm{~B}$ & $6,9 \mathrm{AB}$ & $6,08 \mathrm{~A}$ & $5,66 \mathrm{~A}$ & $\hat{Y}=8,0762-0,7319 x$ & $0,97^{* *}$ \\
\hline$S+E O$ & $9,41 \mathrm{~A}$ & $7,66 \mathrm{~A}$ & $5,78 \mathrm{~A}$ & $4,70 \mathrm{~A}$ & $\hat{Y}=9,8341-1,5111 x$ & $0,99 * *$ \\
\hline$S+S C$ & $7,43 \mathrm{~B}$ & $5,92 \mathrm{~B}$ & $5,62 \mathrm{~A}$ & $5,28 \mathrm{~A}$ & $\hat{Y}=7,328-0,6497 x$ & $0,87^{* *}$ \\
\hline \multicolumn{7}{|c|}{ Diâmetro do Caule $(\mathrm{mm})$} \\
\hline \multirow{2}{*}{ Substratos } & \multicolumn{6}{|c|}{ Salinidade da Água (dS m-1) } \\
\hline & 0,3 & 1,5 & 2,5 & 3,5 & Regressão & $\mathrm{R}^{2}$ \\
\hline S & $1,74 \mathrm{~B}$ & $1,78 \mathrm{AB}$ & $1,72 \mathrm{~A}$ & $1,47 \mathrm{~A}$ & --- & NS \\
\hline$S+E O$ & $2,22 \mathrm{~A}$ & $2,16 \mathrm{~A}$ & $1,72 \mathrm{~A}$ & $1,66 \mathrm{~A}$ & $\hat{Y}=2,3287-0,1991 x$ & $0,87^{* *}$ \\
\hline$S+S C$ & $1,43 \mathrm{~B}$ & $1,38 \mathrm{~B}$ & $1,49 \mathrm{~A}$ & $1,38 \mathrm{~A}$ & --- & NS \\
\hline \multicolumn{7}{|c|}{ Número de Folhas (folha) } \\
\hline \multirow{2}{*}{ Substratos } & \multicolumn{6}{|c|}{ Salinidade da Água (dS $\left.\mathrm{m}^{-1}\right)$} \\
\hline & 0,3 & 1,5 & 2,5 & 3,5 & Regressão & $\mathrm{R}^{2}$ \\
\hline$S$ & $5,42 \mathrm{~B}$ & $5,00 \mathrm{~B}$ & $4,06 \mathrm{~A}$ & $4,00 \mathrm{~A}$ & $\hat{Y}=5,5729-0,4897 x$ & $0,91^{*}$ \\
\hline$S+E O$ & $7,11 \mathrm{~A}$ & $6,83 \mathrm{~A}$ & $4,39 \mathrm{~A}$ & $3,00 \mathrm{~A}$ & $\hat{Y}=8,0157-1,3756 x$ & $0,90 * *$ \\
\hline$S+S C$ & $4,22 \mathrm{~B}$ & $3,33 \mathrm{C}$ & $2,83 \mathrm{~A}$ & $2,67 \mathrm{~A}$ & $\hat{Y}=4,225-0,4929 x$ & $0,93^{*}$ \\
\hline
\end{tabular}


A adição de esterco ovino favoreceu ao maior crescimento das mudas de maracujazeiro, possivelmente em função do maior suprimento nutricional, tendo em vista que esse insumo é de liberação lenta, ao contrário do substrato comercial onde os nutrientes estão prontamente assimiláveis, estando sujeitos à remoção pelo processo de lixiviação dos sais. Os resultados obtidos para AP e NF das mudas produzidas com esterco ovino foram superiores aos obtidos por Almeida et al. (2011) que verificaram altura máxima de $6,53 \mathrm{~cm}$ e 4,40 folhas para as muda cultivadas em substratos comporto por $50 \%$ Solaris $\AA+50 \%$ esterco bovino.

Assim como observado no crescimento vegetativo das mudas o substrato composto por $\mathrm{S}+\mathrm{EO}$, proporcionou o maior acúmulo de matéria seca das mudas de maracujazeiro amarelo, até o nível de salinidade de 1,5 dS $\mathrm{m}^{-1}$, de tal forma que a partir desse nível não foi verificado diferenças significativas entre 0 substratos sob o crescimento das mudas,com exceção para a produção de matéria fresca da parte aérea onde o substrato $S+E O$ também teve superioridade em relação aos demais (Tabela 5).

Observa-se ainda, que as reduções mais acentuadas foram observadas no acúmulo de matéria seca da raiz, reduzindo cerca de $65,8 \%$ quando comparados o acúmulo de matéria seca das plantas sob $0,3 \mathrm{dS} \mathrm{m}^{-1}$ em relação a maior CEa (3,5 dS $\mathrm{m}^{-1}$ ) (Tabela 5).

A redução no crescimento radicular pode estar relacionada ao mecanismo de tolerância da espécie, de modo a reduzir a absorção de água e consequentemente de sais, amenizando a toxicidade por íons específicos (Flowers\&Flowers, 2005) e, desta forma tende a afetar o crescimento e desenvolvimento das distintas partes das plantas. Relatos semelhantes foram verificados em experimento commudas de maracujazeiro amarelo em função do aumento da salinidade da água de irrigação por Cruz et al. (2006).

Redução do acúmulo de matéria seca do sistema radicular em função do aumento da salinidade da água também foi verificado em outras frutíferas, por Brito et al. (2008) e Brito et al. (2014) em porta-enxertos de citros, em Sousa et al. (2011) e Alves et al. (2013) em mudas de cajueiro anão precoce e em Sá et al. (2013) em mudas de mamoeiro.

Tabela 5. Teste de médias e analise de regressão das variáveis matéria seca da parte aérea (MSPA), da raiz (MSR) e total (MST) de mudas de maracujazeiro amarelo em função substratos (SUB) e salinidade da água de irrigação (SAL) aos 60 dias após a semeadura.

\begin{tabular}{|c|c|c|c|c|c|c|}
\hline \multicolumn{7}{|c|}{ Matéria Seca da Parte Aérea (g) } \\
\hline \multirow{2}{*}{ Substrato } & \multicolumn{6}{|c|}{ Salinidade da Água (dS $\left.\mathrm{m}^{-1}\right)$} \\
\hline & 0,3 & 1,5 & 2,5 & 3,5 & Regressão & $R^{2}$ \\
\hline$S$ & $0,14 \mathrm{~B}$ & $0,10 \mathrm{~B}$ & $0,11 \mathrm{~A}$ & $0,07 A B$ & --- & NS \\
\hline$S+E O$ & $0,62 \mathrm{~A}$ & $0,47 \mathrm{~A}$ & $0,15 \mathrm{~A}$ & $0,16 \mathrm{~A}$ & $\hat{Y}=0,6621-0,1606 x$ & $0,88^{* *}$ \\
\hline$S+S C$ & $0,04 \mathrm{~B}$ & $0,05 \mathrm{~B}$ & $0,05 \mathrm{~A}$ & $0,03 \mathrm{C}$ & ---- & NS \\
\hline \multicolumn{7}{|c|}{ Matéria Seca da Raiz (g) } \\
\hline \multirow{2}{*}{ Substrato } & \multicolumn{6}{|c|}{ Salinidade da Água $\left(\mathrm{dS} \mathrm{m}^{-1}\right)$} \\
\hline & 0,3 & 1,5 & 2,5 & 3,5 & Regressão & $\mathrm{R}^{2}$ \\
\hline$S$ & $0,08 \mathrm{~B}$ & $0,05 \mathrm{~B}$ & $0,05 \mathrm{~A}$ & $0,02 \mathrm{~A}$ & --- & NS \\
\hline$S+E O$ & $0,33 \mathrm{~A}$ & $0,20 \mathrm{~A}$ & $0,05 \mathrm{~A}$ & $0,01 \mathrm{~A}$ & $\hat{Y}=0,352-0,1059 x$ & $0,96^{* *}$ \\
\hline$S+S C$ & $0,03 \mathrm{~B}$ & $0,03 \mathrm{~B}$ & $0,02 \mathrm{~A}$ & $0,02 \mathrm{~A}$ & ---- & NS \\
\hline \multicolumn{7}{|c|}{ Matéria Seca Total (g) } \\
\hline \multirow{2}{*}{ Substrato } & \multicolumn{6}{|c|}{ Salinidade da Água (dS m¹) } \\
\hline & 0,3 & 1,5 & 2,5 & 3,5 & Regressão & $\mathrm{R}^{2}$ \\
\hline$S$ & $0,22 \mathrm{~B}$ & $0,15 B$ & $0,16 \mathrm{~A}$ & $0,09 \mathrm{~A}$ & $\hat{Y}=0,2255-0,0363 x$ & $0,90 *$ \\
\hline$S+E O$ & $0,95 \mathrm{~A}$ & $0,67 \mathrm{~A}$ & $0,20 \mathrm{~A}$ & $0,05 \mathrm{~A}$ & $\hat{Y}=1,0141-0,2665 x$ & $0,92^{* *}$ \\
\hline$S+S C$ & $0,06 \mathrm{~B}$ & $0,08 \mathrm{~B}$ & $0,07 \mathrm{~A}$ & $0,05 \mathrm{~A}$ & ---- & NS \\
\hline
\end{tabular}

\section{Conclusões}

O aumento da salinidade da água de irrigação reduziu a emergência, o crescimento e o acúmulo de matéria seca das mudas de maracujazeiro amarelo, sendo a matéria seca total a variável mais afetada pela salinidade.

O substrato contendosolo + esterco ovino na proporção (2:1) promoveu o maior 
crescimento inicial e acúmulo de matéria seca as mudas de maracujazeiro amarelo quando irrigadas com salinidade até o nível de 1,5 dS $\mathrm{m}^{-1}$.

A água salina de até o nível de 1,5 dS $\mathrm{m}^{-1}$, pode ser usada para a produção de mudas de maracujazeiro amarelo.

\section{Referências}

Ahmed, B.A.E., Moritani, I.S. 2010. Effect of saline water irrigation and manure application on the available water. Agricultural Water Management 97(1): 165-170.

Almeida, J.P.N, Barros, G.L, Silva, G.B.P, Procópio, I.J.S, Mendonça, V. 2011. Substratos alternativos na produção de mudas de maracujazeiro amarelo em bandeja. Revista verde de Agroecologia e Desenvolvimento Sustentavel 6(1): 188-195.

Alves, F.A.L., Ponte, L.F.A., Ferreira-Silva, S.L., Maia, J.M., Silveira, J.A.G. 2013. Germinação e estabelecimento de plântulas de cajueiro-anão precoce (AnacardiumoccidentaleL.) em função da salinidade. Revista Brasileira de Ciências Agrárias 8(2):197-204.

Araújo, W.L., Sousa, J.R. M., Sousa Junior, J.R., Silva, S.S., Aleixo, D.L., Pereira, E.B.2013. Produção de mudas de maracujazeiro-amarelo irrigadas com água salina. Agropecuária Científica no Semiárido 9(4): 15-19.

Ayers, R.S., Westcot, D.W. 1999. Qualidade da água na agricultura. Campina Grande: UFPB. 153p. Estudos FAO: Irrigação e Drenagem, 29.

Barnard, J.H., Rensburg, L.D.V., Bennie, A.T.P. 2010.Leaching irrigated saline sandy to sandy loam aped al soils with water of a constant salinity. Irrigation Science 28(2): 191-201.

Brito, M.E.B., Fernandes, P.D., Gheyi, H.R., Melo, A.S., Cardoso, J.A.F., Soares Filho, W.S.2008. Sensibilidade de variedades e híbridos de citrange à salinidade na formação de portaenxertos. Revista Brasileira de Ciências Agrárias 3: 343-353.

Brito, M.E.B., Fernandes, P.D., Gheyi, H.R., Melo, A.S., Soares Filho, W.S., Santos, R.T. 2014. Sensibilidade à salinidade de híbridos trifoliados e outros porta-enxertos de citros. Revista Caatinga $27(1): 17-27$.

Cavalcante, L.F, Silva, G.F, Gheyi, H.R, Dias, T.J, Alves, J.C, Costa, A.P.M. 2009. Crescimento de mudas de maracujazeiro amarelo em solo salino com esterco bovino líquido fermentado. Revista Brasileira de Ciências Agrárias 4(4): 414-420.

Claessen, M.E.C. (Org.). 1997. Manual de métodos de análise de solo. 2.ed. Rev. Atual. Embrapa-CNPS, Rio de Janeiro. Brasil 212p.

Costa, E.C., Leal, P.A.M., Santos, L.C.R., Vieira, L.C.R. 2010. Crescimento de mudas de mamoeiro conduzidas em diferentes ambientes protegidos, recipientes e substratos na região de Aquidauana, Estado do Mato Grosso do Sul. Acta Scientiarum Agronomy 32(3): 463-470.

Cruz, J.L., Pelacani, C.R., Coelho, E.F., Caldas, R.C., Almeida, A.Q., Queiroz, J.R. 2006. Influência da salinidade sobre o crescimento, absorção e distribuição de sódio, cloro e macronutrientes em plântulas de maracujazeiro-amarelo. Bragantia 65: 275-284.

Fernandes, P.D., Brito, M.E.B., Gheyi, H.R., Soares Filho, W.S., Melo, A.S., Carneiro, P.T. 2011. Crescimento de híbridos e variedades portaenxerto de citros sob salinidade. Acta Scientiarum Agronomy 33: 259-267.

Ferreira, D.F. 2011 . Sisvar: a computer statistical analysis system. Ciência Agrotecnologia 35(6): 1039-1042.

Flowers, T.J., Flowers, S.A. 2005. Why does salinity pose such a difficult problem for plant breeders? Agricultural Water Management 78(1): 15-24.

Gurgel, M.T., Gheyi, H.R., Fernandes, P.D., Santos, F.J.S., Nobre, R.G. 2007. Crescimento inicial de porta-enxertos de goiabeira irrigados com águas salinas. Revista Caatinga 20(2): 24-31.

Holanda Filho, R.S.F., Santos, D.B., Azevedo, C.A.V., Coelho, E.F.; Lima, V.L.A. 2011. Água salina nos atributos químicos do solo e no estado nutricional da mandioqueira. Revista Brasileira de Engenharia Agrícola e Ambiental 15(1): 60-66.

IBGE - Instituo Brasileiro de Geografia e Estatística. 2012. Sidra - Produção Agrícola Municipal. http:// www.sidra.ibge.gov.br. <25 Janeiro 2014>

Lima, A.A., Caldas, R.C., Santos, V.S. 2006. Germinação e crescimento de espécies de maracujá. Revista Brasileira de Fruticultura 28: 125-127.

Mendonca, V., Araújo Neto, S.E., Ramos, J.D., Carvalho, J.G., Andrade Junior, V.C. 2006. Fontes e doses de fósforo para o maracujazeiroamarelo. Revista Caatinga 19(1): 65-70.

Pires, A.A., Monnerat, P.H., Marciano, C.B., Pinto, L.G.R., Zampirolli, P.D.,Rosa, R.C.C., Muniz, R.A. 2008. Efeito da adubação Alternativa do maracujazeiro-amarelo nas características químicas e físicas do solo. Revista Brasileira de Ciência do Solo 32(5): 1997-2005.

Sá, F.V.S., Brito, M.E.B., Melo, A.S., Antônio Neto, P., Fernandes, P.D., Ferreira, I.B. 2013. Produção de 
mudas de mamoeiro irrigadas com água salina. Revista Brasileira Engenharia Agrícola Ambiental 17(10): 1047-1054.

Sousa, A.B.O., Bezerra, M.A., Farias, F.C. 2011. Germinação e desenvolvimento inicial de clones de cajueiro comum sob irrigação com água salina. Revista Brasileira de Engenharia Agrícola e Ambiental 15: 390-394.

Sousa, G.B., Cavalcante, L.F., Cavalcante, I.H.L., Beckmann-Cavalcante, M.Z.E Nascimento, J.A. 2008. Salinidade do substrato contendo biofertilizante para formação de mudas de maracujazeiro amarelo irrigado com água salina. Revista Caatinga 21 (2): 172-180.

Souto, A.G.L., Cavalcante, L.F., Nascimento, J.A.M., Mesquita, F.O., Lima Neto, A.J. 2013. Comportamento do noni à salinidade da água de irrigação em solo com biofertilizante bovino. Irriga 18(3): 442-453.

Taiz, L., Zeiger, E. 2013. Fisiologia vegetal. 5.ed. Artmed, Porto Alegre, Brasil. 918p. 